\title{
Handling the pressures of medical practice: Addressing physician stress, burnout, and discontent
}

\author{
Alan H. Rosenstein \\ Consultant in Health Care Behavior Management, Practicing Internist in San Francisco, CA, USA
}

\begin{abstract}
We all went into medicine with the global intent of providing best practice medical care. The years of intense rigorous training, dedication, exhaustion, and debt were all well worth it to enable us to hone our craft and be confident, proud, and respected for what we do. While admittedly some of the expectations and rewards of being a physcian may be influenced by age, generational values, and years of experience, we all want to feel that it was worth the effort and that we still get the pride and joy of being a physician. But the environment has changed and so have the attitudes and behaviors of many physicians
\end{abstract}

\section{Introduction}

Starting with the arrival of "managed care" and its mandated utilization controls and service restrictions more than 35 years ago, physicians first began to experience a concern that outside forces were beginning to intrude on their autonomy and decision making authority when it comes to matters related to patient care. This was perceived as only a minor disturbance in the force and most physicians made the adjustment by extending their willingness to provide "contractual care" to a number of different managed care plans with a variety of acronyms such as HMOs, PPOs, IPA, MSOs, PHOs, IHNs and the like. The joy of practicing medicine still outweighed the secondary external interference [1].

Then starting with the passage of Health Care Reform and Accountable Care, a new series of mandates and initiatives were introduced which had a significant impact on the structure, process, incentives, and priorities of delivering care. This had a much stronger effect on physician attitudes and behaviors toward medical practice. New financial and social accountabilities for a series of quality based performance metrics with increased documentation and reporting requirements, forced compliance with electronic medical records, more publicly available medical information, and changing reimbursement priorities has led to the formation of new full- spectrum models of care focusing on productivity and cost- efficiency which have dramatically changed physician feelings and approaches to care. As a result many physicians moved away from independent solo or group practice into salaried positions in an effort to deal with the pressures and restrictions of the changing health care environment. In response, many physicians (as much as 15\%) chose early retirement [2]. For those that continued it hasn't been a happy landscape. The 2017 Medscape Physician Lifestyle Report revealed that more than $65 \%$ of physicians reported dissatisfaction with work. [3] In a comprehensive national survey the Mayo Clinic indicated that more than $50 \%$ of physicians reported high levels of stress and burnout [4]. With a looming physician shortage this presents both an individual physician and overall society concern [5, 6]. So what can and should we do?

\section{Consequences}

There is definitely a call for action. Besides the issue of access to care, there is a growing amount of literature suggesting that unhappy, frustrated, stressed, and/ or burnout physicians can lead to compromises in patient safety, quality, and overall satisfaction with outcomes of care [7-9]. The major concern is that physicians are either unaware of how these issues are affecting their practice, and/ or are unwilling to do what's necessary in an effort to improve the situation.

\section{Barriers}

The first barrier is physician awareness. Many physicians are unware of the effect that stress may have on both their physical and emotional well- being. Even if they are aware they look at stress as necessary evil for doing business. The second barrier is acknowledgement. After all physicians are used to working under a demanding high pressure sleep deprived environment, so what's new? The third barrier is ego. Physicians have very strong egocentric personalities which makes them reluctant to seek or accept outside advice or support. Concerns about what others may think about their competency and fears of confidentiality also inhibit their willingness to take action. The final barrier is the time or motivation for change. Physicians are so overwhelmed that it is difficult for them to think about taking time away from practice and think they can take care of everything themselves.

\section{Strategies}

This is a complex situation. The overall strategy needs to focus primarily on what the benefit is to the individual physician rather than for the organizational good. Support programs need to be sensitive to the physician's time and priorities, assure confidentiality, and work to support efficiencies in the process and flow to enhance medical delivery.

Correspondence to: Alan H. Rosenstein, Consultant in Health Care Behavior Management, Practicing Internist in San Francisco, CA, USA, Tel: 415370 7754; E-mail:ahrosensteinmd@aol.com

Received: April 20, 2017; Accepted: May 02, 2017; Published: May 05, 2017 
Physicians need help but the problem is that they won't seek it on their own. Therefore, we need to look to the organizations in which the physician is associated with to take a pro-active role in trying to help physicians better adjust to the pressures of today's healthcare environment.

Support can come through a variety of channels (Table 1). On the logistical/ administrative end organizations need to be sensitive to physician workloads and capacity. Adjusting on-call responsibilities, easing productivity requirements, limiting non-clinical responsibilities, and/ or providing additional support to assist in electronic medical documentation and reporting will all help free up time for the physician to focus on clinical responsibilities. Clinical support can be offered through the utilization of Nurse Practitioners, Physician Assistants, and/ or Care Managers who can ease some of the routine clinical responsibilities and enable the physician to spend more time on complex cases. Third, and most important, is to provide emotional/ behavioral support services.

Working with the physcian to teach them how to work more effectively in today's fast paced health care environment will provide a constructive process to enhance efficiency, gratification, and satisfaction with care delivery. This will require a multidimensional approach.

At one end of the spectrum organizations should provide education and training programs on such topics as stress management, conflict management, and process management to help reduce or improve outcomes in difficult situations. A second approach is to improve their overall staff and patient relationship skills. Training programs in emotional intelligence, diversity management, communication skills development, team collaboration skills, and customer service can all improve work relationships which will improve efficiencies in information flow, set clear expectations, and improve overall task compliance. A third approach is to provide individual coaching or counseling.

An individualized approach will get the greatest benefits. Using services available through Physician Wellness Committees, Physician Employee Assistance Programs (EAPs), or through individualized coaching (either through internal or outsourced resources) will enable strategies that can adopted to address specific concerns and needs.

One of the key underlying essentials is the underlying organizational culture. Many recent studies have stressed the importance of strong organizational leadership commitment and investment in resource support in positively enhancing the attitudes and performance of the physcian staff [10].

As mentioned previously, all these services should be designed to help the physician learn techniques to help them adapt more successfully to the pressures of today's medical practice.

Table 1: Strategic Recommendations
\begin{tabular}{|c|}
\hline 1. Address physician barriers \\
\hline Time/ interest/ confidentiality/motivation \\
\hline 2. Support services: \\
\hline • Administrative \\
\hline - Clinical \\
\hline 3. Organizational culture \\
\hline 4. Physcian engagement \\
\hline 5. Physician Well- being \\
\hline 6. Physician enthusiasm \\
\hline
\end{tabular}

The next strategy is to improve overall physcian engagement [11]. In order to get physicians to take action the physician needs to feel like you understand their world and are responsive to their individual needs and concerns. Engagement starts by giving the physician an opportunity for input and discussion. Input can be gathered though Town Hall meetings, Department meetings, specific task forces, or better yet, though one on one discussion sessions. Giving the physician a voice and an opportunity to express their concerns is often the first step in reducing their frustration, anger, and stress. The next step is to be responsive to their needs. It's not that you have to solve all of their problems, but at least you can show empathy and build trust and respect around developing mutually agreeable goals and collegial relationships that focus on the physician's role and responsibilities that promote patient centric care.

The final step is promote physician well- being $[12,13]$. There is a growing amount of research focusing on the importance of physical and emotional well- being and its affect on physician satisfaction, engagement, and care relationships, all of which lead to improved outcomes of care. Many organizations are now trying to improve physician well-being by offering appropriate health and wellness programs and activities and providing enhanced relaxation programs focusing on mindfulness and resilience training. The over-riding factor of course is to get the physician to make it a priority to set aside the time for rest and relaxation.

We all need to work together to restore the physician's compassion for medical care. We need to remind them of purpose and reward, and treat them with respect, recognition, and admiration for what they do [14].

\section{Conclusion}

There are many studies suggesting that physician burnout is on the verge of being a health care crisis [15]. Physicians are a precious resource and we need to do what we can to help them better adjust to the pressures and complexities of today's medical practice. But we can't leave it up to the physician to take the initiative on their own. So, we need to look to the organizations in which the physician is associated with to take a pro-active role in providing encouragement and support, and to do it before a crisis occurs. Understand the physician world, listen to what they have to say, show empathy and understanding, provide appropriate individualized support, and teach them the skills to enhance care relationships that will make their life easier, improve overall efficiency and satisfaction, and return joy to their world.

\section{References}

1. Rosenstein A (2017) The Impact of Stress, Burnout, and Personality on Physician Attitudes and Behaviors that Impact Patient Care. Psychology and Behavioral Science International Journal 3: 1-3.

2. VITAL WorkLife \& Cejka Search (2015) Physician Stress and Burnout Survey

3. Medscape Lifestyle Report (2017) Race and Ethnicity, Bias and Burnout

4. Shanafelt TD, Hasan O, Dyrbye LN, Sinsky C, Satele D, et al. (2015) Changes in Burnout and Satisfaction With Work-Life Balance in Physicians and the General US Working Population Between 2011 and 2014. Mayo Clin Proc 90: 1600-1613. [Crossref]

5. Bernstein L (2015) U.S. Faces 90,000 Doctor Shortage by 2025, Medical School Association Warns.

6. AAMC (2017) Physician Supply and Demand Through 2025: Key Findings

7. Salyers M, Bonfils K, Luther L, Firmin R, et al. (2017) The Relationship Between Professional Burnout and Quality and Safety in Healthcare: A Meta-Analysis. Journal of General Internal Medicine 32: 475-482. [Crossref] 
8. Danielson D, Ketterling R, Rosenstein A (2013) Physician Stress and Burnout: Causes, Effects, and Impact on Performance and Behavior. AMGA Group Practice Journal 62: $38-41$

9. Privitera M, Rosenstein A, Plessow F, LoCastro T (2014) Physician Burnout and Occupational Stress: An Inconvenient Truth with Unintended Consequences. Journal of Hospital Administration 4: 27-35.

10. Shanafelt TD1, Gorringe G2, Menaker R3, Storz KA4, Reeves D4, et al. (2015) Impact of organizational leadership on physician burnout and satisfaction. Mayo Clin Proc 90: 432-440. [Crossref]

11. Rosenstein A (2015) Strategies to Enhance Physician Engagement. Journal of Medical Practice Management 31: 113-116. [Crossref]
12. West C, Dyrbye L, Rabatin J, Call T (2014) Intervention to Promote Physician WellBeing, Job Satisfaction, and Professionalism: A Randomized Clinical Trial. JAMA Internal Medicine 174: 527-533. [Crossref]

13. Rosenstein A (2016) Understanding the Psychology Behind Physician Attitudes, Behaviors, and Engagement as the Pathway to Physician Well- Being. Journal of Psychology and Clinical Psychiatry 5: 1-4

14. Jager A, Tutty M, Kao A (2017) Association Between Physician Burnout and Identification with Medicine as a Calling. Mayo Clinic Proceedings 92: 415-422.

15. Noseworthy J, Madara J, Cosgrove D, Edgeworth M (2017) Physician Burnout is a Public Health Care Crisis.

Copyright: $(02017$ Rosenstein AH. This is an open-access article distributed under the terms of the Creative Commons Attribution License, which permits unrestricted use, distribution, and reproduction in any medium, provided the original author and source are credited. 